\title{
The relationship between sarcopenia and survival at 1 year in patients having elective colorectal cancer surgery
}

\author{
D. R. Dolan ${ }^{1} \cdot$ K. A. Knight ${ }^{2} \cdot$ S. Maguire ${ }^{1} \cdot$ S. J. Moug ${ }^{2}$
}

Received: 24 May 2019 / Accepted: 20 August 2019 / Published online: 5 September 2019

(c) The Author(s) 2019

\begin{abstract}
Background Colorectal cancer remains a common cause of cancer death in the UK, with surgery being the mainstay of treatment. An objective measurement of the suitability of each patient for surgery, and their risk-benefit calculation, would be of great utility. We postulate that sarcopenia (low muscle mass) could fulfil this role as a prognostic indicator. The aim of this study was to determine the relationship between sarcopenia and long-term outcomes in patients undergoing elective bowel resection for colorectal cancer.

Methods One hundred and sixty-three consecutive patients who had elective curative colorectal resection for cancer were eligible for inclusion in the study. Psoas muscle mass was assessed on preoperative computed tomography scan at the level of the L3 vertebra and standardised for patient height (total psoas index, TPI). Sarcopenia (low muscle mass) was defined as $<524 \mathrm{~mm}^{2} / \mathrm{m}^{2}$ in males and $385 \mathrm{~mm}^{2} / \mathrm{m}^{2}$ in females. In addition to clinical-pathological parameters, postoperative complications were recorded and patients were followed up for mortality for 1 year after surgery.

Results Sarcopenia was present in $19.6 \%$ of the study participants and was significantly related to body mass index $(p=0.007), 30$-day mortality $(p=0.042)$ and 1-year mortality $(p=0.046)$. In univariate analysis, American Society of Anesthesiologists grade $(p=0.016)$, tumour stage $(p=0.018)$ and sarcopenia $(p=0.043)$ were found to be significant independent predictors of 1-year mortality.

Conclusions This study has found sarcopenia to be prevalent in patients with colorectal cancer having elective surgery. Independent of age, sarcopenia was associated with poorer 30-day mortality and survival at 1 year. Measurement of muscle mass preoperatively could be used to stratify a patient's risk, allowing targeted strategies such as prehabilitation, to be implemented to modify sarcopenia and improve long-term outcomes for patients.
\end{abstract}

Keywords Colorectal cancer $\cdot$ Sarcopenia $\cdot$ One-year survival

\section{Introduction}

Colorectal cancer is the fourth most common cancer in the UK, with over 41,000 new diagnoses in 2014 [1]. With incidence increasing with age, and the ageing population

Accepted for presentation as a poster at: National Cancer Research Institute Conference 2018, Glasgow.

Accepted for presentation as a poster at: European Society of Surgical Research 2019, Geneva.

S. J. Moug

susanmoug@nhs.net

1 School of Medicine, University of Glasgow, Glasgow, UK

2 Department of Surgery, Royal Alexandra Hospital, Corsebar Road, Paisley PA2 9PN, UK expanding, there is a clinical need for new prognostic knowledge-aided decision making to improve long-term outcomes $[2,3]$. One potential prognostic marker is sarcopenia. Sarcopenia was defined in 2010 as 'a syndrome characterised by progressive and generalised loss of skeletal muscle mass and strength' (the European Working Group on Sarcopenia in Older People (EWGSOP) [4]. There are various techniques available to measure sarcopenia, varying from simple to complex, but computed tomography (CT) measurement of the psoas muscle, with established cutoffs defining sarcopenia, is a widely accepted technique [4].

There have been numerous studies that have assessed the relationship between sarcopenia and surgical outcomes in patients having various intra-abdominal operations, including radical cystectomy, liver transplantation and emergency 
general surgery, with their findings pointing towards an association between sarcopenia and mortality [5-8].

Studies specifically analysing the influence of sarcopenia on postoperative elective colorectal cancer patients have found that the presence of sarcopenia results in an increased risk of postoperative complications, increased length of hospital stay and increased cost of care [9-15]. A study published in 2019 showed that sarcopenia is highly predictive of serious postoperative complications in colorectal cancer patients [15]. In contrast, there are only a few studies focusing on the relationship between sarcopenia and long-term mortality [16-19]. Although all suggested a negative influence of sarcopenia on 1-year survival, drawing conclusions is difficult due to the different patient populations studied and varying methodologies.

The primary aim of this study was to determine the relationship between preoperative sarcopenia and mortality at 1 year in patients who had elective colorectal cancer resection.

\section{Materials and methods}

\section{Data sources and study population}

The study was registered with the NHS Greater Glasgow and Clyde Clinical Effectiveness Department (August 2016). Analysis was undertaken of data extracted from the prospective enhanced recovery after surgery (ERAS) database at the Royal Alexandra Hospital, Paisley, for the period January 2015-December 2016. This database has been set up as a part of the National Enhanced Recovery Colorectal Initiative (NERCI) supported by the Whole System Patient Flow Improvement Programme as part of the Scottish Government's Health Performance and Delivery Directorate. Briefly, data from all elective colorectal surgery (both benign and malignant pathology) are submitted from each surgical unit in Scotland every month with regular feedback after central analysis of the data.

Inclusion criteria extended to any patient who had elective colorectal resection with curative intent, where pathology had confirmed colonic or rectal adenocarcinoma. Stage 4 patients with resectable liver and lung metastases, as adjudged by the relevant clinical specialists, were included. Patients were excluded if: they had received neo-adjuvant therapy; had surgery to treat any condition other than colorectal cancer; had widespread unresectable metastatic disease; had surgery as an emergency; if no preoperative CT scan was available; or if their most recent CT scan was performed more than 4 months before surgery.

Demographic, anthropometric and clinical data including height, weight, body mass index (BMI) [weight $(\mathrm{kg}) /$ height $\left.(\mathrm{m})^{2}\right]$, American Society of Anesthesiologists (ASA) score, operative procedure, American Joint Committee on Cancer (AJCC) TNM stage [20], length of hospital stay (days), postoperative complications and grade [21] and re-admission to the hospital within 30 days were analysed. In addition, patients' electronic records were followed up for mortality for 1 year after the date of surgery.

\section{Sarcopenia measurement: CT scan analysis}

To measure sarcopenia in each patient, the total cross-sectional area of the psoas muscles (total psoas area, TPA) was measured using a manual technique at the level of the L3 vertebra on preoperative $\mathrm{CT}$ (median interval between $\mathrm{CT}$ scan and surgery was 38 days, range $2-119$ days) [22]. To ensure standardisation, the exact level of measurement was defined as the CT slice in which both L3 transverse processes were maximally in view. Area was measured using a free-hand drawing technique on Picture Archiving and Communication System (PACS) software (Fig. 1). The outline of each individual psoas muscle was traced, the area of each calculated, and summated to provide the TPA $\left(\mathrm{mm}^{2}\right)$. The TPA was then standardised for patient height using the formula: TPA $\left(\mathrm{mm}^{2}\right) /$ height $\left(\mathrm{m}^{2}\right)$. This provided the total psoas index (TPI) for each patient.

For the purposes of this study, the threshold values used for the diagnosis of sarcopenia are the same as those used by Prado et al. in their widely cited 2008 paper [23]: $524 \mathrm{~mm}^{2} /$ $\mathrm{m}^{2}$ for males and $385 \mathrm{~mm}^{2} / \mathrm{m}^{2}$ for females. All individuals with a TPI below this threshold for their gender were classified as sarcopenic. For the purposes of this study, sarcopenia is defined as an absolute variable; patients were either sarcopenic or non-sarcopenic.

To ensure reliability of our technique, 20 scans were randomly selected and measured for TPI by blindly trained investigators to allow calculation of inter- and intra-class correlation coefficients (ICCC). The ICCC values for interand intra-class reliability were 0.94 and 0.99 , respectively (values close to 1 indicate excellent agreement).

\section{Statistical analysis}

Relevant variables were transcribed to SPSS version 21 [24] to enable statistical analysis. Descriptive statistics were used to characterise the patient population, with continuous variables summarised by median and interquartile range and categorical variables presented in tabulated form with percentages. Continuous variables were tested using nonparametric methods.

To determine the primary outcome of the influence of sarcopenia on survival at 1 year, survival analysis using log-rank testing was performed. Further exploration of variables influencing mortality was performed using univariate 


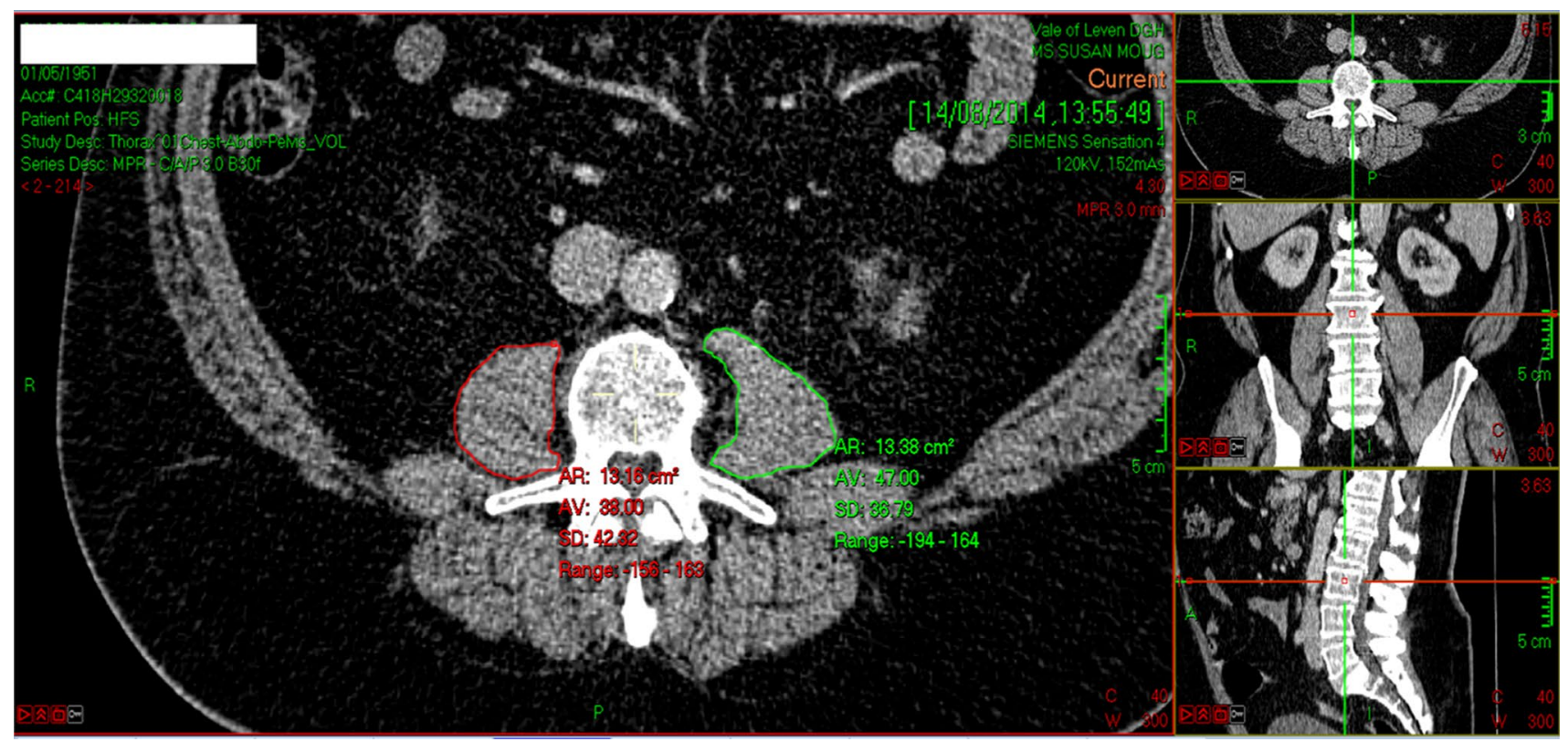

Fig. 1 CT image at the level of the L3 vertebra, demonstrating the method of outlining the left (green) and right (red) psoas muscle and measuring their respective areas

analysis and a Cox proportional hazards model. The level of significance was set at $5 \%$.

\section{Results}

\section{Patient demographics, physiological and pathological characteristics}

Over the study period, 331 patients had elective colorectal surgery, with 163 (49.2\%) eligible for inclusion in the study (Fig. 2). The median age of the cohort was 70 years (IQR 61-75) and 99 (60.7\%) were male. The majority of surgery was for patients with rectal cancer who had an ASA of 2 and $112(68.7 \%)$ were overweight or obese. One hundred and forty-nine (91.4\%) had a pathological $\mathrm{R} 0$ resection with only $8.6 \%$ of patients having a major complication leading to a median length of the hospital stay of 8 days (IQR 6-12) [Table 1]. Eleven patients had stage 4 tumours, however, these patients had lung/liver metastases that were considered resectable and so they were operated on with curative intent.

\section{Sarcopenia}

Overall, 32 (19.6\%) patients having elective colorectal cancer surgery were sarcopenic: 18/99 males (18.2\%) (Fig. 3) and 14/64 females (21.9\%) (Figs. 3, 4). The characteristics of the sarcopenic and non-sarcopenic groups are compared in Table 2. Significant differences were noted between the two groups in the categories: BMI $(p=0.007), 30$-day mortality $(p=0.042)$ and 1 -year mortality $(p=0.046)$. Here, the sarcopenic patients were more likely to be classified as underweight and have increased postoperative mortality at 30 days and 1 year [Fig. 5].

\section{Survival at 1 year}

For the whole patient cohort, univariate analysis found sarcopenia $(p=0.043)$, tumour stage $(p=0.018)$, ASA grade $(p=0.016)$ and major complications $(p=0.021)$ to be significantly associated with survival at 1 year. Multivariable analysis revealed that only ASA grade and tumour stage were significantly independent predictors of mortality at 1 year $(p=0.042$ and $p=0.007$, respectively) (Table 3 ).

\section{Discussion}

This study demonstrated that sarcopenia is prevalent, occurring in nearly one-fifth of the patients having elective colorectal cancer surgery. In addition to confirming that sarcopenia places patients at greater risk of postoperative complications, this work has found sarcopenia also negatively influences survival at 1 year after curative surgery.

These results have strengths over the previous publications looking at 1 year survival. It is prospective and contains both colon and rectal cancer patient populations, not just rectal [18]. In addition, two of the previous studies assessed sarcopenia in Asian populations, and members of these populations have accepted differences in lifestyle and 


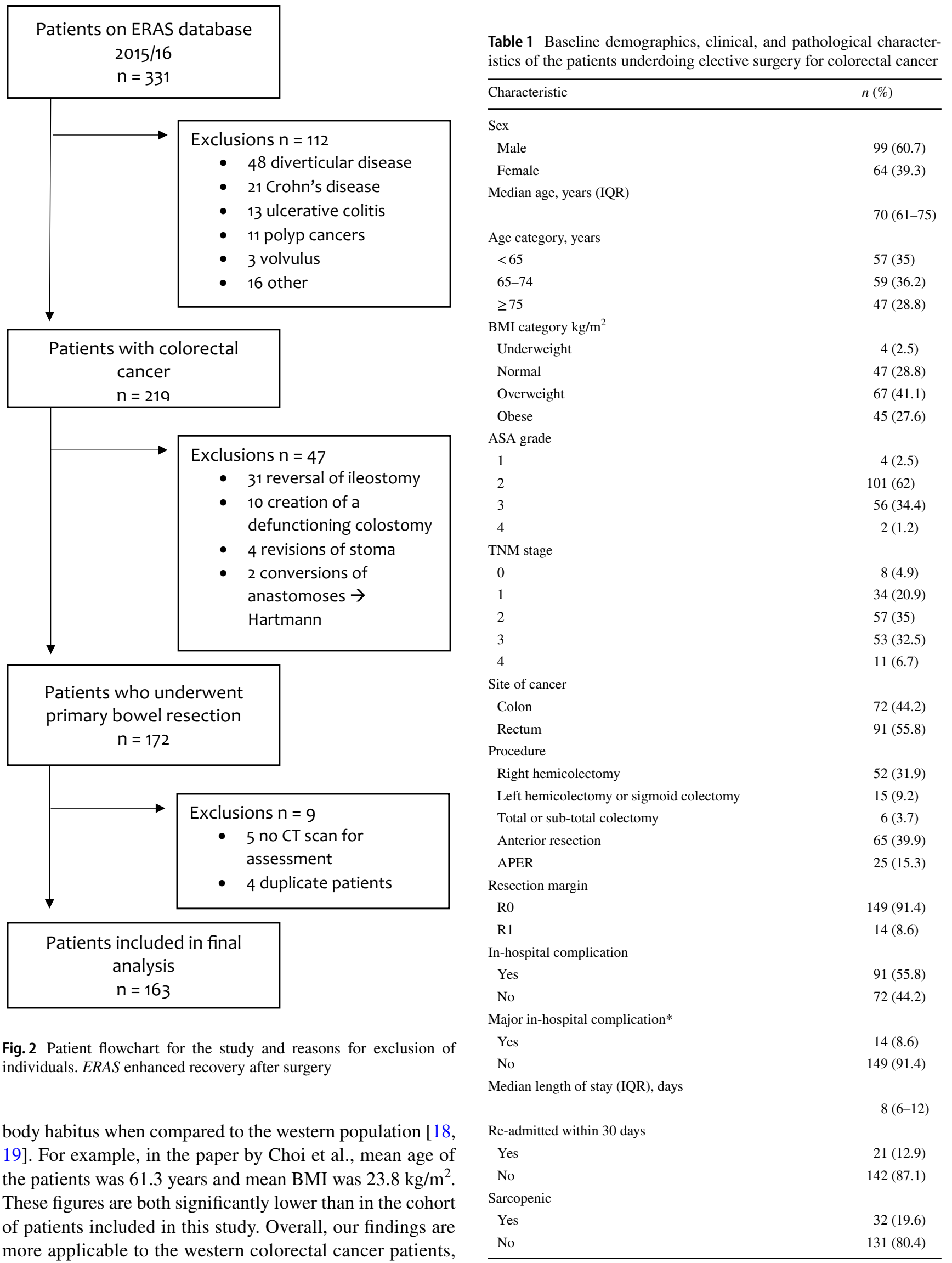


Table 1 (continued)

*Complications classified as 3 or greater on the Clavien-Dindo scale ASA American Society of Anesthesiologists, AJCCTNM American Joint Committee on Cancer TNM stage, BMI body mass index adding to the evolving evidence that sarcopenia is a negative prognostic factor for patients having elective gastrointestinal
Fig. 3 Scatterplot showing the spread of TPI values for male patients and the threshold for sarcopenia

Fig. 4 Scatterplot showing the spread of TPI values for female patients and the threshold for sarcopenia
TPI and Sarcopenia Threshold- Male
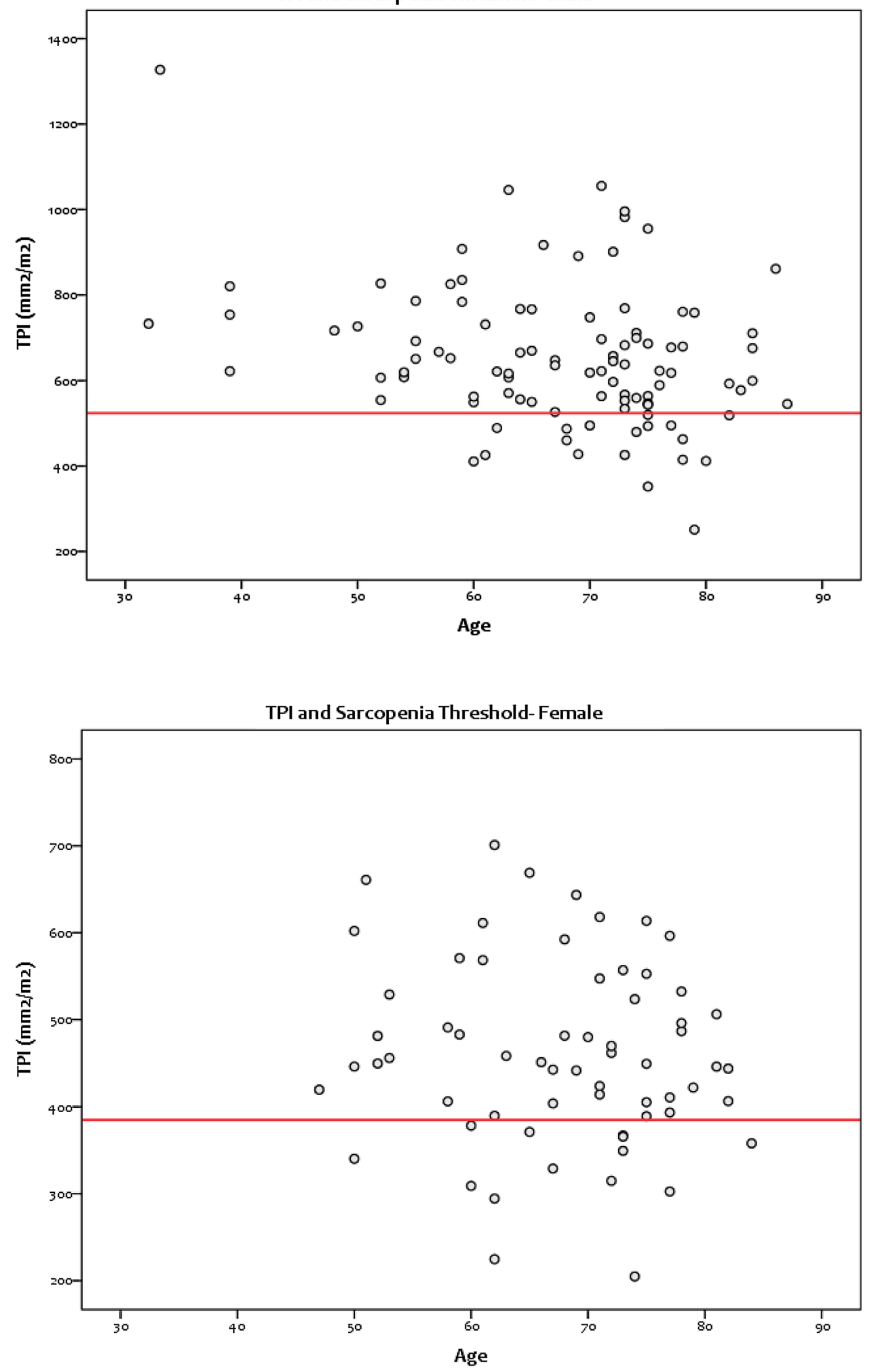
Table 2 Demographics, clinical, and pathological characteristics of patients with sarcopenia compared to patients without sarcopenia

\begin{tabular}{|c|c|c|c|}
\hline & Sarcopenic $(n=32)$ & $\begin{array}{l}\text { Non-sarcopenic } \\
(n=131)\end{array}$ & $p$ value \\
\hline \multicolumn{4}{|l|}{ Sex } \\
\hline Male & $18(56.3)$ & $81(61.8)$ & \multirow[t]{2}{*}{0.350} \\
\hline Female & $14(43.8)$ & $50(38.2)$ & \\
\hline \multicolumn{4}{|c|}{ Age category, years } \\
\hline$<65$ & $8(25.0)$ & $49(37.4)$ & \multirow[t]{3}{*}{0.412} \\
\hline $65-74$ & $13(40.6)$ & $46(35.1)$ & \\
\hline$\geq 75$ & $11(34.4)$ & $36(27.5)$ & \\
\hline \multicolumn{4}{|c|}{ BMI category $\mathrm{kg} / \mathrm{m}^{2}$} \\
\hline Underweight & $3(9.4)$ & $1(0.8)$ & \multirow[t]{4}{*}{$0.007 *$} \\
\hline Normal & 13 (40.6) & $34(26.0)$ & \\
\hline Overweight & $11(34.4)$ & $56(42.7)$ & \\
\hline Obese & $5(15.6)$ & $40(30.5)$ & \\
\hline \multicolumn{4}{|c|}{ Median ASA (SD) } \\
\hline & $2(0.56)$ & $2(0.55)$ & 0.542 \\
\hline \multicolumn{4}{|l|}{ TNM stage } \\
\hline 0 & $3(9.4)$ & $5(3.8)$ & \multirow[t]{5}{*}{0.194} \\
\hline 1 & $3(9.4)$ & $31(23.7)$ & \\
\hline 2 & $12(37.5)$ & $45(34.4)$ & \\
\hline 3 & $12(37.5)$ & $41(31.3)$ & \\
\hline 4 & $2(6.3)$ & $9(6.9)$ & \\
\hline \multicolumn{4}{|l|}{ Site of cancer } \\
\hline Colon & $17(53.1)$ & $55(42.0)$ & \multirow[t]{2}{*}{0.174} \\
\hline Rectum & $15(46.9)$ & $76(58.0)$ & \\
\hline \multicolumn{4}{|c|}{ Resection margin } \\
\hline R0 & $30(93.8)$ & $119(90.8)$ & \multirow[t]{2}{*}{0.598} \\
\hline R1 & $2(6.3)$ & $12(9.2)$ & \\
\hline \multicolumn{4}{|c|}{ In-hospital complication } \\
\hline Yes & $14(43.8)$ & $73(55.7)$ & \multirow[t]{2}{*}{0.957} \\
\hline No & $18(56.3)$ & $60(44.3)$ & \\
\hline \multicolumn{4}{|c|}{ Major in-hospital complication } \\
\hline Yes & $2(6.3)$ & $12(9.2)$ & \multirow[t]{2}{*}{0.598} \\
\hline No & $30(93.8)$ & $119(90.8)$ & \\
\hline \multicolumn{4}{|c|}{ Median length of stay (IQR), days } \\
\hline & $8(6-12)$ & $8(6-12)$ & 0.567 \\
\hline \multicolumn{4}{|c|}{ Re-admitted within 30 days } \\
\hline Yes & $3(9.4)$ & $18(13.7)$ & \multirow[t]{2}{*}{0.509} \\
\hline No & $28(90.6)$ & $113(86.3)$ & \\
\hline \multicolumn{4}{|l|}{ Alive at 30 days } \\
\hline Yes & $31(96.9)$ & $131(100)$ & \multirow[t]{2}{*}{$0.042 *$} \\
\hline No & $1(3.1)$ & $0(0)$ & \\
\hline \multicolumn{4}{|c|}{ Alive at 90 days } \\
\hline Yes & $31(96.9)$ & $130(0.8)$ & \multirow[t]{2}{*}{0.277} \\
\hline No & $1(3.1)$ & $1(99.2)$ & \\
\hline Alive at 1 year & & & \\
\hline Yes & $27(84.4)$ & $124(94.7)$ & $0.046^{*}$ \\
\hline No & $5(15.6)$ & $7(5.3)$ & \\
\hline
\end{tabular}

Complications classified as 3 or greater on the Clavien-Dindo scale

ASA American Society of Anesthesiologists, AJCCTNM American Joint Committee on Cancer TNM stage, $B M I$ body mass index

$* P<0.05$ : level of significance oncological surgery, and more specifically, colorectal resection.

The mechanism underlying the significant relationship between sarcopenia and 1-year mortality requires discussion. It may be indicative of the presence of sub-clinical synchronous metastasis, which is subsequently picked up during follow-up after surgery. If this is the case, then sarcopenia may highlight patients who would benefit from more extensive staging investigations, such as positron emission tomography (PET) scanning. This may lead to the consideration of preoperative chemotherapy and/or more frequent clinical and radiological follow-up with the aim of improving outcomes. An alternative explanation is that sarcopenia indicates that patients have poorer physical capacity, making them less resilient to the physiological stresses of surgery and more at risk of complication [15]. Indeed, the relationship between sarcopenia and frailty is well documented [25]. This raises the possibility that, unlike other preoperative prognostic markers (e.g. TNM staging), sarcopenia could be modified via prehabilitation. This individualised physical activity-centred intervention is delivered in the period between diagnosis and the commencement of treatment for cancer and has been shown to be feasible, safe and reduce postoperative complications in patients treated for colorectal cancer [26, 27], although may not always produce improvements in fitness. Furthermore, a recent systematic review has demonstrated that the positive effects of prehabilitation can be seen after as little as 2 weeks [28]. Therefore, the pretreatment measurement of sarcopenia could offer an effective way of selecting patients who would benefit the most from such targeted, individualised prehabilitation [29]. Individualised prehabilitation programmes will require adequate funding to introduce; however, we do not expect interventions to be particularly costly, and would hope that the institution of prehabilitation would lead to savings in resources due to reduced morbidity and shortened stays in hospital.

The strengths of our study were that the data were collected prospectively and are reviewed centrally on a monthly basis, minimising the likelihood of any inaccuracy in the measurement or recording of variables. The method used to measure sarcopenia is simple and easy to learn, low cost, valid, and requires no investment from healthcare providers, meaning it could be seamlessly integrated into the care pathway for colorectal cancer patients. This is in contrast to other methods of skeletal muscle measurement that use costly specialist software packages that require additional training.

It is important to acknowledge several limitations of this work. First, this is a single-centre study and future work should be multi-centred to allow greater number of patients with colorectal cancer to be included. A multi-centre study would also allow the inclusion of a more ethnically diverse population and subsequent subgroup analysis. Second, 
Fig. 5 Comparison of 1-year mortality between colorectal cancer patients with sarcopenia and those without sarcopenia

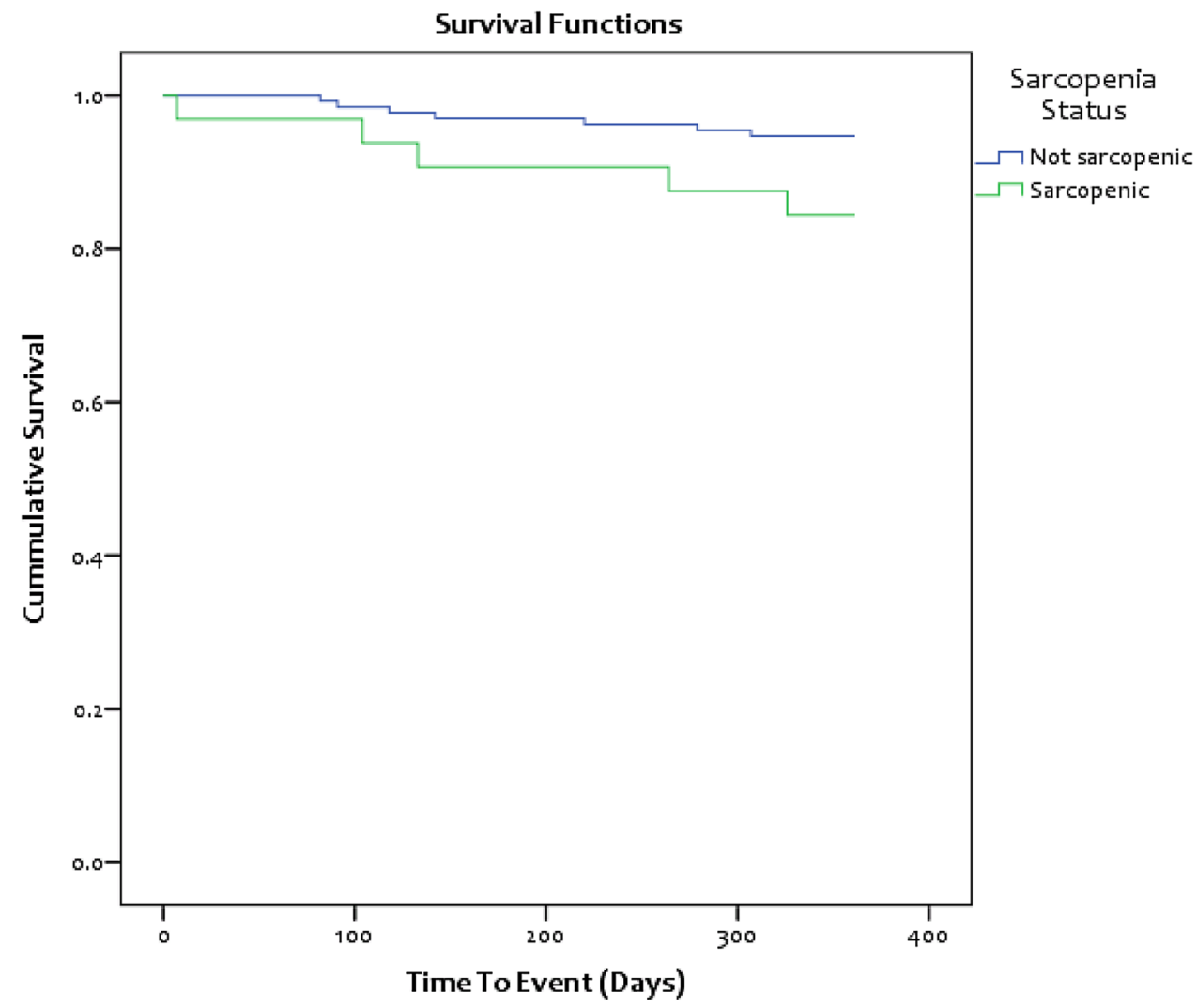

Table 3 Univariate and multivariable analyses of factors influencing survival at 1 year in patients who have undergone elective surgery for colorectal cancer

\begin{tabular}{lllll}
\hline & \multicolumn{2}{l}{$\begin{array}{l}\text { Univariate analysis } \\
\text { Variable }\end{array}$} & \multicolumn{2}{l}{ Multivariable analysis $^{\mathrm{a}}$} \\
\cline { 3 - 5 } & Log-rank $p$ value & Hazard ratio & $95 \%$ CI & $p$ value \\
\hline Sarcopenia & 0.043 & 2.233 & $0.665-7.504$ & 0.194 \\
TNM stage & 0.018 & 2.609 & $1.307-5.208$ & 0.007 \\
ASA & 0.016 & 2.861 & $1.037-7.892$ & 0.042 \\
Major complication & 0.021 & - & - & - \\
BMI category & 0.225 & - & - & - \\
Age group & 0.546 & - & - & - \\
Gender & 0.418 & - & - & - \\
\hline
\end{tabular}

ASA American Society of Anesthesiologists, BMI body mass index

${ }^{a}$ Included preoperative variables that had been identified as significant in univariate analysis although all scans were performed within 4 months of surgery, it is possible that the skeletal muscle mass could have changed before surgery. Furthermore, the use of predefined cutoffs for sarcopenia has been defined in populations, which are not necessarily homogenous to the population included in this study. For example, the Prado sarcopenia cutoffs used in this study were defined in an obese population in Canada, with tumours of the respiratory and gastrointestinal tracts. However, using predefined cutoffs is preferable to arbitrarily concluding that patients in the lowest quartile for muscle mass are sarcopenic. Lastly, 30-day mortality was related to sarcopenia in this study. However, this finding was driven by a single death in the cohort and should be interpreted with caution.

\section{Conclusions}

This study has found sarcopenia to be prevalent in colorectal cancer patients having elective surgery, resulting in poorer long-term survival. CT measurement of total psoas mass is a valid and simple technique for diagnosing sarcopenia that could be used to augment existing methods of patient-risk stratification prior to surgery. Such sarcopenic patients could then undergo targeted strategies such 
as prehabilitation, to improve both their short- and longterm outcomes.

Acknowledgements We gratefully acknowledge support from The Carnegie Trust of Scotland who awarded the lead author a Vacation Scholarship to allow completion of this work.

Author contributions SJM conceived and designed the study. DRD and KAK co-ordinated the data collection. SM and DRD performed the sarcopenia analysis. DRD, KAK and SJM interpreted the data. All the authors revised manuscript drafts, approved the final manuscript, and contributed intellectually important content.

Funding This study received no funding.

\section{Compliance with ethical standards}

Conflict of interest All the authors declare no conflicts of interest and have completed the ICMJE uniform disclosure form at www.icmje .org/coi_disclosure.pdf.

Ethical approval No additional ethical approval was required. The National Patient Flow Database is registered with the Scottish Government with Caldicott approval.

Informed consent For this study informed consented was not needed.

Transparency declaration The corresponding author affirms that this manuscript is an honest, accurate, and transparent account of the study being reported; that no important aspects of the study have been omitted; and that any discrepancies from the study as planned (and, if relevant, registered) have been explained.

Open Access This article is distributed under the terms of the Creative Commons Attribution 4.0 International License (http://creativeco mmons.org/licenses/by/4.0/), which permits unrestricted use, distribution, and reproduction in any medium, provided you give appropriate credit to the original author(s) and the source, provide a link to the Creative Commons license, and indicate if changes were made.

\section{References}

1. Cancer Research UK. Statistics by cancer type. http://www.cance rresearchuk.org/health-professional/cancer-statistics/statistics-bycancer-type/bowel-cancer. Accessed 30 Nov 2017

2. Ait Ouakrim D, Pizot C, Boniol M, Malvezzi M, Boniol M, Negri E et al (2015) Trends in colorectal cancer mortality in Europe: retrospective analysis of the WHO mortality database. BMJ 351:h4970. https://doi.org/10.1136/bmj.h4970

3. Office for National Statistics. What does the 2011 Census tell us about older people? 6th September 2013. https://www.ons.gov.uk/ peoplepopulationandcommunity/birthsdeathsandmarriages/agein g/articles/whatdoesthe2011 censustellusaboutolderpeople/201309-06. Accessed 04 Mar 2019

4. Ruz-Jentoft AJ, Baeyens JP, Bauer JM et al (2010) Sarcopenia: European consensus on definition and diagnosis: report of the European Working Group on sarcopenia in older people. Age Ageing 39(4):412-423. https://doi.org/10.1093/ageing/afq034

5. Ahmadi H, Montie JE, Weizer AZ et al (2015) Patient psoas muscle mass as a predictor of complications and survival after radical cystectomy. Curr Urol Rep 16:79. https://doi.org/10.1007/s1193 4-015-0548-0

6. Gani F, Buettner S, Margonis GA, Sasaki K, Wagner D, Kim Y, Hundt J, Kamel IR, Pawlik TM (2016) Sarcopenia predicts costs among patients undergoing major abdominal operations. Surgery 160(5):1162-1171. https://doi.org/10.1016/j.surg.2016.05.002 (ISSN 0039-6060)

7. Englesbe MJ, Patel SP, He K et al (2010) Sarcopenia and postliver transplant mortality. J Am Coll Surg 211(2):271-278. https ://doi.org/10.1016/j.jamcollsurg.2010.03.039

8. Yang D, Karvellas CJ, Baracos V, Williams DC, Khadaroo RG (2014) Sarcopenia is a predictor of outcomes in very elderly patients undergoing emergency surgery. Surgery 156(3):521-527. https://doi.org/10.1016/j.surg.2014.04.027 (ISSN 0039-6060)

9. Lieffers JR, Bathe OF, Fassbender K, Winget M, Baracos VE (2012) Sarcopenia is associated with postoperative infection and delayed recovery from colorectal cancer resection surgery. Br J Cancer 107:931-936. https://doi.org/10.1038/bjc.2012.350

10. Reisinger KW, van Vugt JL, Tegels J, Snijders C, Hulsewe KW, Hoofwijk AG et al (2015) Functional compromise reflected by sarcopenia, frailty, and nutritional depletion predicts adverse postoperative outcome after colorectal cancer surgery. Ann Surg 261(2):345-352. https://doi.org/10.1097/sla.0000000000000628

11. Huang DD, Wang SL, Zhuang CL, Zheng BS, Lu JX, Chen FF et al (2015) Sarcopenia, as defined by low muscle mass, strength and physical performance, predicts complications after surgery for colorectal cancer. Colorectal Dis 17(11):O256-O264. https:// doi.org/10.1111/codi.13067

12. Reisinger KW, Derikx JP, van Vugt JL, Von Meyenfeldt MF, Hulsewe KW, Olde Damink SW et al (2016) Sarcopenia is associated with an increased inflammatory response to surgery in colorectal cancer. Clin Nutr 35(4):924-927. https://doi.org/10.1016/j. clnesp.2018.01.003

13. Nakanishi R, Oki E, Sasaki S, Hirose K, Jogo T, Edahiro K, Korehisa S, Taniguchi D, Kudo K, Kurashige J, Sugiyama M, Nakashima Y, Ohgaki K, Saeki H, Maehara Y (2018) Sarcopenia is an independent predictor of complications after colorectal cancer surgery. Surg Today 48(2):151-157. https://doi.org/10.1007/ s00595-017-1564-0

14. Gani F, Buettner S, Margonis GA, Sasaki K, Wagner D, Kim Y, Hundt J, Kamel IR, Pawlik TM (2016) Sarcopenia predicts costs among patients undergoing major abdominal operations. Surgery 160(5):1162-1171

15. Herrod PJJ, Boyd-Carson H, Doleman B, Trotter J, Schlichtemeier S, Sathanapally G, Somerville J, Williams JP, Lund JN (2019) Quick and simple; psoas density measurement is an independent predictor of anastomotic leak and other complications after colorectal resection. Tech Coloproctol 23(2):129-134. https://doi. org/10.1007/s10151-019-1928-0

16. Malietzis G, Currie A, Athanasiou T, Johns N, Anyamene N, Glynne-Jones R et al (2016) Influence of body composition profile on outcomes following colorectal cancer surgery. Br J Surg 103(5):572-580

17. Miyamoto Y, Baba Y, Sakamoto Y, Ohuchi M, Tokunaga R, Kurashige J et al (2015) Sarcopenia is a negative prognostic factor after curative resection of colorectal cancer. Ann Surg Oncol 22(8):2663-2668

18. Choi M, Oh S, Lee I, Oh S, Won D (2017) Sarcopenia is negatively associated with long-term outcomes in locally advanced rectal cancer. J Cachexia Sarcopenia Muscle 9(1):53-59

19. Hopkins J, Reif R, Bigam D, Baracos V, Eurich D, Sawyer M (2019) The impact of muscle and adipose tissue on long-term survival in patients with stage I to III colorectal cancer. Dis Colon Rectum 62(5):549-560

20. https://www.cancer.org/cancer/colon-rectal-cancer/detectiondiagnosis-staging/staged.html. Accessed 17 Apr 2018 
21. Dindo D, Demartines N, Clavien P-A (2004) Classification of surgical complications: a new proposal with evaluation in a cohort of 6336 patients and results of a survey. Ann Surg 240(2):205-213

22. Jones KI, Doleman B, Scott S, Lund JN, Williams JP (2015) Simple psoas cross-sectional area measurement is a quick and easy method to assess sarcopenia and predicts major surgical complications. Colorectal Dis 17:O20-O26. https://doi.org/10.1111/ codi. 12805

23. Prado C, Lieffers J, McCargar L, Reiman T, Sawyer M, Martin $\mathrm{L}$ et al (2008) Prevalence and clinical implications of sarcopenic obesity in patients with solid tumours of the respiratory and gastrointestinal tracts: a population-based study. Lancet Oncol 9(7):629-635

24. American Cancer Society. AJCC staging of colorectal cancer. https://www-01.ibm.com/support/docview.wss?uid=swg2160806 0. Accessed 17 Apr 2018

25. Cesari M, Landi F, Vellas B, Bernabei R (2014) Marzetti E. Two sides of the same coin. frontiers in aging neuroscience, Sarcopenia Phys Frailty, p 6

26. Moug S, Mutrie N, Barry S, Mackay G, Steele R, Boachie C et al (2019) Prehabilitation is feasible in patients with rectal cancer undergoing neoadjuvant chemoradiotherapy and may minimize physical deterioration: results from the REx trial. Colorectal Dis 21(5):548-562

27. Barberan-Garcia A, Ubré M, Roca J, Lacy A, Burgos F, Risco $\mathrm{R}$ et al (2018) Personalised prehabilitation in high-risk patients undergoing elective major abdominal surgery. Ann Surg 267(1):50-56

28. Faithfull S, Turner L, Poole K, Joy M, Manders R, Weprin J et al (2019) Prehabilitation for adults diagnosed with cancer: a systematic review of long-term physical function, nutrition and patientreported outcomes. Eur J Cancer Care (Engl) 28(4):e13023

29. Buffart L, Sweegers M, May A, Chinapaw M, van Vulpen J, Newton $\mathrm{R}$ et al (2018) Targeting exercise interventions to patients with cancer in need: an individual patient data meta-analysis. J Natl Cancer Inst 110(11):1190-1200

Publisher's Note Springer Nature remains neutral with regard to jurisdictional claims in published maps and institutional affiliations. 